\title{
miR-199b-5p inhibits triple negative breast cancer cell proliferation, migration and invasion by targeting DDR1
}

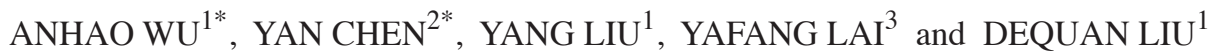 \\ ${ }^{1}$ Department of Breast Surgery Ward I, ${ }^{2}$ Cancer Institute, The Third Affiliated Hospital, \\ Kunming Medical University, Tumor Hospital of Yunnan Kunming Province, Kunming, Yunnan 650100; \\ ${ }^{3}$ Public Health, Guizhou Medical University, Guiyang, Guizhou 550004, P.R. China
}

Received November 1, 2017; Accepted July 2, 2018

DOI: $10.3892 / \mathrm{ol} .2018 .9255$

\begin{abstract}
Triple negative breast cancer (TNBC) has received increasing attention from oncologists worldwide due to its poor prognosis and paucity of targeted therapies. MicroRNAs (miRs) are a group of small non-coding RNAs that are responsible for the post-transcriptional regulation of various target genes. The present study demonstrated that the expression of miR-199b-5p in breast cancer tissue was significantly reduced compared with that in normal breast tissues by reverse transcription-quantitative polymerase chain reaction. In addition, western blot analysis and luciferase reporter assays revealed that miR-199b-5p in TNBC cells inhibited discoidin domain receptor tyrosine kinase 1 expression by directly targeting its 3'-untranslated region. Furthermore, miR-199b-5p markedly suppressed the proliferation and invasion of TNBC cells, as demonstrated by using wound-healing, migration, invasion and proliferation assays. Collectively, these results indicate that miR-199b-5p may be a novel alternative therapeutic target for TNBC.
\end{abstract}

\section{Introduction}

MicroRNAs (miRNAs and miRs) are a group of endogenous small non-coding RNAs, which cause the degradation or translational repression of its target mRNA by binding to it and inhibiting the expression of its target gene (1). It has been demonstrated that abnormal expression of miR-199b-5p is associated with various diseases and certain processes, including tumorigenesis, tumor progression, invasion and metastasis (2-7). In previous studies, $\mathrm{miR}-199 \mathrm{~b}-5 \mathrm{p}$ has been revealed to be closely

Correspondence to: Dr Dequan Liu, Department of Breast Surgery Ward I, The Third Affiliated Hospital, Kunming Medical University, Tumor Hospital of Yunnan Province, 519 Kunzhou Road, Kunming, Yunnan 650100, P.R. China

E-mail: gaoligongshan@sohu.com

${ }^{*}$ Contributed equally

Key words: microRNA, discoidin domain receptor tyrosine kinase 1, microRNA-199b-5p, triple negative breast cancer associated with the prognosis of patients with osteosarcoma and overall and disease-free survival rates were significantly lower in patients exhibiting high levels of miR-199b-5p expression compared with those exhibiting low levels. It was also confirmed to serve a primary role in the regulation of proliferation, migration and invasion of osteosarcoma cells in vivo (2). Additionally, Fang et al (3) demonstrated that miR-199b-5p inhibits the proliferation, migration and clonogenicity of HER2+ breast cancer cell lines by directly targeting HER2. It was also demonstrated that downregulation of miR-199b-5p expression was associated with poor prognosis in patients with breast cancer (4). Triple-negative breast cancer (TNBC) is aggressive and is associated with higher rates of metastasis and poorer survival times than other breast cancer subtypes (8). Furthermore, a lack of effective therapy poses a significant challenge to physicians (8). However, to the best of our knowledge, the role of miR-199b-5p in TNBC has not been assessed. miR-199b-5p can be used as a diagnostic marker and a novel therapeutic target for various types of cancer, and has significant clinical application value $(2,5-7)$. Therefore, it is important to further investigate the role of miR-199b-5p in TNBC. Discoidin domain receptor tyrosine kinase 1 (DDR1) is a class of collagen receptor that is overexpressed in a variety of epithelial malignancies, including ovarian, breast, endometrial, esophageal and lung cancer (9-19). A previous study demonstrated that a DDR $1^{\text {Low }} / \mathrm{DDR} 2^{\text {High }}$ protein profile is associated with TNBC and may identify patients with invasive carcinoma with a poor prognosis (20). High DDR1 expression may also serve a role in breast cancer cell proliferation and/or survival $(21,22)$.

To the best of our knowledge, the present study is the first to demonstrate that miR-199b-5p inhibits the proliferation, migration and invasion of TNBC cells. It was also revealed that miR-199b-5p inhibited DDR1 gene expression by directly targeting its $3^{\prime}$ untranslated region (UTR). These results indicate that miR-199b-5p may be a novel alternate therapeutic target for TNBC. In terms of clinical samples, the expression of miR-199b-5p was significantly reduced in breast cancer tissues compared with normal breast tissue.

\section{Materials and methods}

Patients and specimen collection. The present study was approved by the Ethics Committee of Kunming Medical 
University (The Third Affiliated Hospital of Kunming Medical University, Kunming, China) and informed consent was obtained from all patients. Breast cancer tissue and normal tissue ( $>5 \mathrm{~cm}$ distant from cancer tissue) were collected from patients who were pathologically diagnosed with breast cancer at the Tumor Hospital of Yunnan Province from December 2015 to March 2016. None of the patients underwent any therapy prior to specimen collection, including chemotherapy and radiotherapy. Collected tissue samples were immediately stored in RNAlater (Tiangen Biotech Co., Ltd., Beijing, China) at $-80^{\circ} \mathrm{C}$. A total of 19 female patients with breast cancer were recruited into the present study (age range, 39-68 years; mean average age, 53 years). The clinicopathological characteristics of each patient were recorded, including age, tumor size, Tumor-Node-Metastasis (TNM) stage, estrogen receptor (ER) status, progesterone receptor (PR) status and lymph node involvement.

Cell lines and cell culture. The human triple negative breast cancer cell line, HCC1937, and the mammary gland cell line, MCF-10A, were donated by Professor Ceshi Chen from the Kunming Institute of Zoology (Kunming, China), CAS (Chinese Academy of Sciences). was recovered from cryopreservation in liquid nitrogen $\left(-196^{\circ} \mathrm{C}\right)$ and used at an early passage. 293 cells were purchased from the American Type Culture Collection (Manassas, VA, USA). HCC1937 cells were maintained in RPMI-1640 medium (HyClone; GE Healthcare Life Sciences, Logan, UT, USA) supplemented with $10 \%$ fetal bovine serum (FBS; HyClone; GE Healthcare Life Sciences), $10 \mathrm{U} / \mathrm{ml}$ penicillin and $0.1 \mathrm{mg} / \mathrm{ml}$ streptomycin (HyClone; GE Healthcare Life Sciences). MCF-10A cells were maintained in Dulbecco's Modified Eagle's medium (DMEM)-F12 medium (HyClone; GE Healthcare Life Sciences) supplemented with $10 \% \mathrm{FBS}, 10 \mathrm{U} / \mathrm{ml}$ penicillin and $0.1 \mathrm{mg} / \mathrm{ml}$ streptomycin. 293 cells were maintained in Dulbecco's Modified Eagle's medium (DMEM; HyClone; GE Healthcare Life Sciences) supplemented with $10 \% \mathrm{FBS}, 10 \mathrm{U} / \mathrm{ml}$ penicillin and $0.1 \mathrm{mg} / \mathrm{ml}$ streptomycin. HCC1937, MCF-10A and 293 cells were then incubated at $37^{\circ} \mathrm{C}$ in $5 \% \mathrm{CO}_{2}$.

$R N A$ isolation and reverse transcription quantitative-polymerase chain reaction ( $R T-q P C R)$. Tissue miRNA was extracted using the miRcute miRNA isolation kit (Tiangen Biotech Co., Ltd.), according to the manufacturer's protocol. cDNA was synthesized using the miRcute Plus miRNA First-strand cDNA Synthesis kit (Tiangen Biotech Co., Ltd.). The thermocycling conditions were as follows: $60 \mathrm{~min}$ at $42^{\circ} \mathrm{C}$ and $3 \mathrm{~min}$ at $95^{\circ} \mathrm{C}$. miR-199b-5p expression was analyzed using the miRcute Plus miRNA qPCR Detection kit (Tiangen Biotech Co., Ltd.) with the 7500 Real-Time PCR system (Applied Biosystems; Thermo Fisher Scientific, Inc., Waltham, MA, USA). The thermocycling conditions were as follows: An initial pre-denaturation step of $15 \mathrm{~min}$ at $95^{\circ} \mathrm{C}$, followed by 40 cycles of denaturation at $94^{\circ} \mathrm{C}$ for $20 \mathrm{sec}$ and annealing at $60^{\circ} \mathrm{C}$ for $34 \mathrm{sec}$. The primers sequences are as follows miR-199b-5p, forward, 5'-CAGCCCAGTGTTTAGACT ATC-3' and reverse, 5'-CAGTGCAGGGTCCGAGGT-3', and U6, forward, 5'-CTCGCTTCGGCAGCACATATACT-3' and reverse, 5'-ACGCTTCACGAATTTGCGTGTC-3'. Relative expression was calculated using the $2^{-\Delta \Delta C q}$ method (23). The
RT-qPCR assays were performed in duplicate and the data were presented as the mean \pm standard error of the mean. Using SPSS 13.0 software (SPSS, Inc., Chicago, IL, USA) for statistical analysis, the relative quantity (RQ) value was defined as the ratio of miR-199b-5p expression in target samples to that in control samples. An RQ $<1$ and $\geq 1$ indicated low and high expression of miR-199b-5p in the target samples, respectively.

Transient transfection of miRNA mimic. The miR-199b-5p mimic and negative control (NC) miRNA were purchased from Shanghai GenePharma Co., Ltd. (Shanghai, China). The miR-199b mimics sequence was 5'-CCCAGUGUUUAG ACUAUCUGUUC-3' and the NC sequence was 5'-UUCUCC GAACGUGUCACGUTT-3'. The miR-199b-5p mimic and $\mathrm{NC}(40 \mathrm{nM})$ were transfected into cells using Lipofectamine RNAiMAX (Invitrogen; Thermo Fisher Scientific, Inc.) in serum-free conditions for $6 \mathrm{~h}$ prior to replacement with complete medium. Western blotting and RT-qPCR were performed $48 \mathrm{~h}$ post-transfection.

Luciferase reporter assay. To assess the mechanism by which miR-199b-5p inhibits cell proliferation and migration, an miRNA target search was performed using TargetScan (http://www.targetscan.org). The results demonstrated that the 3'UTR of DDR1 contained a highly conserved putative miR-199b-5p binding site. Luciferase reporter plasmids, pRL-DDR1-3'UTR wild type (WT) and pRL-DDR1-3'UTR mutant (MUT) plasmids were synthesized and verified by Shanghai Genomeditech Co., Ltd (Shanghai, China). For the luciferase reporter assay, 293 cells were co-transfected with pRL-DDR1-3'UTR WT or pRL-DDR1-3'UTR MUT, and miR-199b-5p mimics or NC using Lipofectamine 2000 (Invitrogen; Thermo Fisher Scientific, Inc.). The pRL-TK plasmid (Promega Corporation, Madison, WI, USA) was used as control. The successful overexpression vector was incubated with LB (Luria-Bertani) liquid medium overnight at $37^{\circ} \mathrm{C}$ and the plasmid was prepared using a high purity Plasmid Extraction kit (Qiagen GmbH, Hilden, Germany), according to the manufacturer's instructions. The pRL-TK 3'-UTR target plasmid (10 ng) was transiently transfected into 293 cells, using Lipofectamine 2000 (Invitrogen; Thermo Fisher Scientific, Inc.) with a pRL-TK 3'-UTR negative control plasmid (10 ng). Results were obtained according to the protocol of the reportable gene detection kit (Shanghai Genomeditech Co., Ltd., Shanghai, China) and luciferase activities were analyzed using a Dual Luciferase Reporter assay system (Promega Corporation, Madison, WI, USA) $48 \mathrm{~h}$ after transfection. Firefly luciferase activity was used for normalization.

Western blot analysis. Total protein from cells was extracted using 1\% radioimmunoprecipitation lysis buffer (Beijing Solarbio Science \& Technology Co., Ltd., Beijing, China) containing phenylmethanesulfonylfluoride (Beijing Solarbio Science \& Technology Co., Ltd.). The miR-199b-5p mimic group was transfected with miR-199b-5p mimics, the negative control group transfected with negative control miR, the control group was untreated. The protein concentration of total cell lysates was measured using a BCA protein assay kit (Tiangen Biotech Co., Ltd.). Proteins (20 $\mu \mathrm{g})$ were separated by $10 \%$ SDS-PAGE and transferred to polyvinylidene 
fluoride membranes (EMD Millipore, Billerica, MD, USA). Membranes were blocked at room temperature with 5\% non-fat milk for $1 \mathrm{~h}$ and incubated at $4{ }^{\circ} \mathrm{C}$ overnight with the following antibodies: DDR1 (dilution, 1:500; cat. no.ENT1307; Elabscience, Wuhan, China) and GAPDH (dilution, 1:3,000; cat. no. 21612; Signalway Antibody LLC, College Park, MD, USA). Membranes were then washed with TBST three times for $10 \mathrm{~min}$, incubated with a goat anti-rabbit immunoglobulin G secondary antibody (dilution, 1:3,000; cat. no. L3012; Signalway Antibody LLC) for $2 \mathrm{~h}$ at room temperature and washed with Tris-buffered saline with Tween 3 times for $10 \mathrm{~min}$. The protein was detected by chemiluminescence using an ECL detection reagent (Beijing Solarbio Science \& Technology Co., Ltd.). Finally, the quantification of western blotting was performed using Image $\mathrm{J}$ software (version 1.51; National Institutes of Health, Bethesda, MD, USA).

Wound-healing assay. HCC1937 cells were transfected with the miR-199b-5p mimic and negative control for $48 \mathrm{~h}$. A marker pen and a ruler were first used to draw 5 lines on each well of a 6-well culture dish. Subsequently, $2 \times 10^{6} \mathrm{HCC} 1937$ cells cultured for $12 \mathrm{~h}$ at $37^{\circ} \mathrm{C}$ in $5 \% \mathrm{CO}_{2}$, and then a $200-\mu \mathrm{l}$ pipette tip was used to scratch two or more parallel wounds of $\sim 200 \mathrm{~mm}$ in width, perpendicular to the marker lines. Wounds were observed following incubation at $37^{\circ} \mathrm{C}$ in $5 \% \mathrm{CO}_{2}$ for 12 and $24 \mathrm{~h}$ under a phase contrast microscope (magnification, $\mathrm{x} 100)$ to assess healing rate.

Cell migration and invasion assays. The miR-199b-5p mimic and negative control were transfected into HCC1937 cells as aforementioned. Transwell chambers without and with Matrigel (Corning Incorporated, Corning, NY, USA) were used to assess cell migration and invasion, respectively. A total of $5 \times 10^{4}$ cells in $200 \mu 1$ RPMI-1640 medium without FBS were placed into the cell culture inserts (BD Biosciences Japan, Co., Ltd, Tokyo, Japan). A total of $600 \mu 1$ RPMI-1640 medium containing $10 \%$ FBS was added to the bottom chamber. Cells were then incubated for $24 \mathrm{~h}$ at $37^{\circ} \mathrm{C}$ in $5 \% \mathrm{CO}_{2}$, fixed with paraformaldehyde and stained with $0.1 \%$ crystal violet at room temperature for $20 \mathrm{~min}$. Cell migration and invasion were analyzed by counting the cells on the underside of the membrane using a light microscope (magnification, x100) in 5 random fields of view.

Cell proliferation assay. The effect of miR-199b-5p on the proliferation of HCC1937 cells was assessed using a CCK-8 assay. Cells in $200 \mu$ l of DMEM were seeded into 96-well plates $\left(2 \times 10^{3} /\right.$ well) and incubated at $37^{\circ} \mathrm{C}$ in $5 \% \mathrm{CO}_{2}$ for $48 \mathrm{~h}$. Cell proliferation was evaluated every $12 \mathrm{~h}$ for 1 day. A total of $10 \mu \mathrm{l} \mathrm{CCK-8} \mathrm{(Biosharp,} \mathrm{Hefei,} \mathrm{China)} \mathrm{was} \mathrm{added} \mathrm{to} \mathrm{each}$ well and incubated for an additional $4 \mathrm{~h}$ at $37^{\circ} \mathrm{C}$ in $5 \% \mathrm{CO}_{2}$. A CCK8 Enzyme-linked immune monitor (Thermo Fisher Scientific, Inc.) was utilized to assess absorbance values at a wavelength of $450 \mathrm{~nm}$.

Statistical analyses. Statistical analysis was performed using SPSS 13.0 software (SPSS, Inc., Chicago, IL, USA). Data are presented as the mean \pm standard error of the mean and were analyzed using a Student's t-test (between two groups) or one-way analysis of variance followed by Tukey's test (multiple groups). The expression of miR-199b-5p and clinicopathological parameters were analyzed using a $\chi^{2}$ test. $\mathrm{P}<0.05$ was considered to indicate a statistically significant difference. Statistical analyses were performed using GraphPad Prism 7.0a Macintosh Version (GraphPad Software Inc., La Jolla, CA, USA).

\section{Results}

miR-199b-5p promotes cell migration and invasion. The TNBC cell line, HCC1937, exhibited the lower expression of miR-199b-5p compared with the MCF-10A cell line (Fig. 1A). To determine the effect of miR-199b-5p on HCC1937 cell migration and invasion, cells were treated with a miR-199b-5p mimic and negative control miR. Observation with fluorescence microscope (Fig. 1B) and the results of RT-qPCR assay (Fig. 1C) demonstrated that the relative expression of miR-199b-5p was significantly elevated in the mimic group compared with the negative control. A wound-healing assay demonstrated that the miR-199b-5p mimic significantly decreased the migration rate of HCC1937 cells (Fig. 2A) compared with the negative control. Furthermore, the Transwell migration assay revealed that miR-199b-5p mimics significantly decreased the number of migrated cells compared with the negative control group. The effect of the miR-199b-5p mimic on HCC1937 cell invasion was assessed using a Matrigel invasion assay, which demonstrated similar results to the migration assay (Fig. 2B).

miR-199b-5p inhibits HCC1937 cell proliferation in vitro. To assess the role of miR-199b-5p in the proliferation of HCC1937 cells, a gain-of-function approach was utilized. HCC1937 cells were transfected with a miR-199b-5p mimic and negative control miR. Furthermore, cell proliferation was evaluated using a CCK- 8 assay at different time points. It was revealed that the upregulation of miR-199b-5p significantly inhibited HCC1937 cell proliferation at days 3-5 in comparison with the control group (Fig. 3A).

The expression of miR-199b-5p in breast cancer tissue is significantly reduced compared with normal breast tissue. A total of 19 samples were obtained from patients with breast cancer who did not receive any treatment prior to surgery. Breast cancer tissues and normal adjacent breast tissue $(>5 \mathrm{~cm}$ from the lesion) were excised. Compared with normal breast tissues, the expression of miR-199b-5p in breast cancer tissues was significantly decreased (Fig. 3B). Furthermore, there was no significant association between miR-199b-5p expression and any assessed characteristics, including age, tumor size and TNM stage were observed (Table I).

The expression of miR-199b-5p is negatively associated with DDR1 in breast cancer tissues. The expression of miR-199b-5p and DDR1 in breast cancer and the association between them were analyzed using public databases (http://starbase.sysu. edu.cn). Compared with normal breast tissue, the expression of miR-199b-5p in breast cancer tissues was significantly reduced (Fig. 4A). These results are congruent with those that were obtained from the present study. Furthermore, compared with normal breast tissue, the expression of DDR1 in breast cancer tissues was significantly upregulated (Fig. 4B). The 


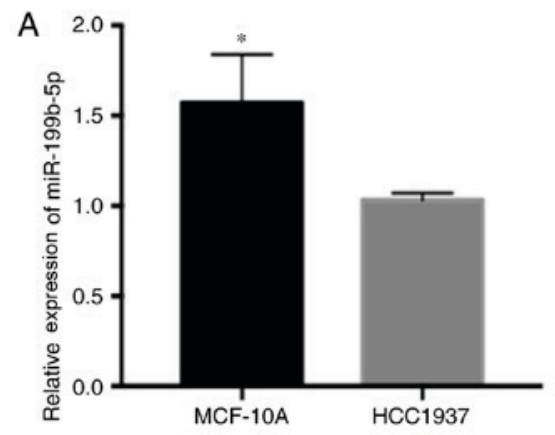

C

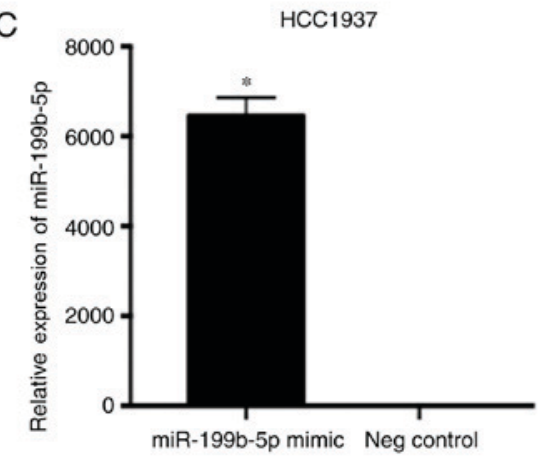

B

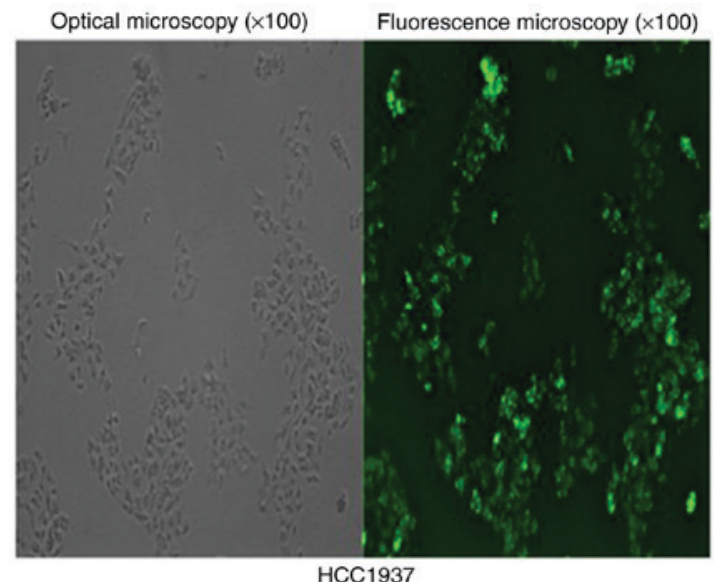

D

DDR1 3'UTR I..5'AGAAAUAUAGgaUAGACACUGGA.

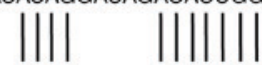

Hsa-miR-199b-5p 3'CUUGUCUAUCAGAUUUGUGACCC

Figure 1. Expression levels of miR-199b-5p in HCC1937 and MCF-10A cell lines and evaluation of the efficiency of HCC1937-cell transfection, and possible target genes of miR-199b-5p were forecasted by bioinformatics tools. (A) The expression of miR-199b-5p was assessed in different breast cell lines (MCF-10A and HCC1937). (B) The expression of GFP in the cells was observed by fluorescence microscope following $12 \mathrm{~h}$ after transfection, the numbers of positive expression cells in every high power field (x100) were counted. (C) Following transfection with the miR-199b-5p mimic, the expression of miR-199b-5p in breast cancer cells was increased, as indicated by a reverse transcription quantitative polymerase chain reaction assay. (D) The TargetScan alignment of DDR1's 3'UTR with hsa-miR-199b-5p indicating the highly conserved regions. "P<0.05 vs. HCC1937 cells and the negative control group. miR, microRNA; DDR1, discoidin domain receptor tyrosine kinase 1; UTR, untranslated region; neg, negative; hsa, homo sapiens.

expression of miR-199b-5p was also demonstrated to be negatively correlated to DDR1 (Fig. 4C).

$D D R 1$ is a candidate target of $m i R-199 b-5 p$. To assess the mechanism by which miR-199b-5p inhibits cell proliferation and migration, an miRNA target search was performed using TargetScan (http://www.targetscan.org). The results demonstrated that the 3'UTR of DDR1 contained a highly conserved putative miR-199b-5p binding site. Western blotting also revealed that the expression of DDR1 was significantly decreased in the miR-199b-5p mimic group compared with the negative control group and the control group (Fig. 5A). In addition, pRL-DDR1 3'UTR-wild type (WT) and DDR1 pRL-3'UTR-mutant (MUT) plasmids were generated to assess the interaction between miR-199b-5p and its putative target sequences. The results of the luciferase reporter assay demonstrated that the luciferase activity of DDR1 3'UTR-luc-WT was decreased upon transfection with the miR-199b-5p mimic, compared with those transfected with negative control (Fig. 5B). These results indicated that miR-199b-5p directly inhibited DDR 1 expression by binding to the 3'UTR of DDR1.

Taken together, the data of the present study indicate that miR-199b-5p serves as a potent oncogenic miRNA in HCC1937 cells by affecting cell proliferation, migration and invasion, possibly by targeting DDR1.

\section{Discussion}

MiRNA is a non-coding RNA comprised of 19-24 nucleotides in eukaryotes, whose primary function is to regulate the expression of target genes (24). According to a previous study, $60-70 \%$ of human and other mammalian genes are regulated by miRNAs (25). In several types of cancer, including hepatocellular carcinoma, renal cell carcinoma, osteosarcoma and acute myeloid leukemia, miR-199b-5p has been revealed to be a biomarker $(2,5-7)$. A further study demonstrated that the downregulation of miR-199b-5p is associated with poor prognosis in patients with breast cancer (4). The study also revealed that miR-199b-5p inhibited HER2+ breast cancer cell proliferation, migration and clonogenicity by directly targeting HER2 (3). However, the role of miR-199b-5p in TNBC has not yet been assessed. The present study demonstrated that different levels of miR-199b-5p were exhibited in different cells. The TNBC cell line HCC1937 exhibited the lower expression of miR-199b-5p compared with the MCF-10A cell line. To assess the effect of miR-199b-5p on the physiological processes of TNBC cells, CCK-8, wound-healing and Transwell assays were performed. The results of these assays indicated that the overexpression of miR-199b-5p inhibited breast cancer cell proliferation, migration and invasion.

The expression of miR-199b-5p in breast cancer and normal breast tissue was detected using RT-qPCR and the 

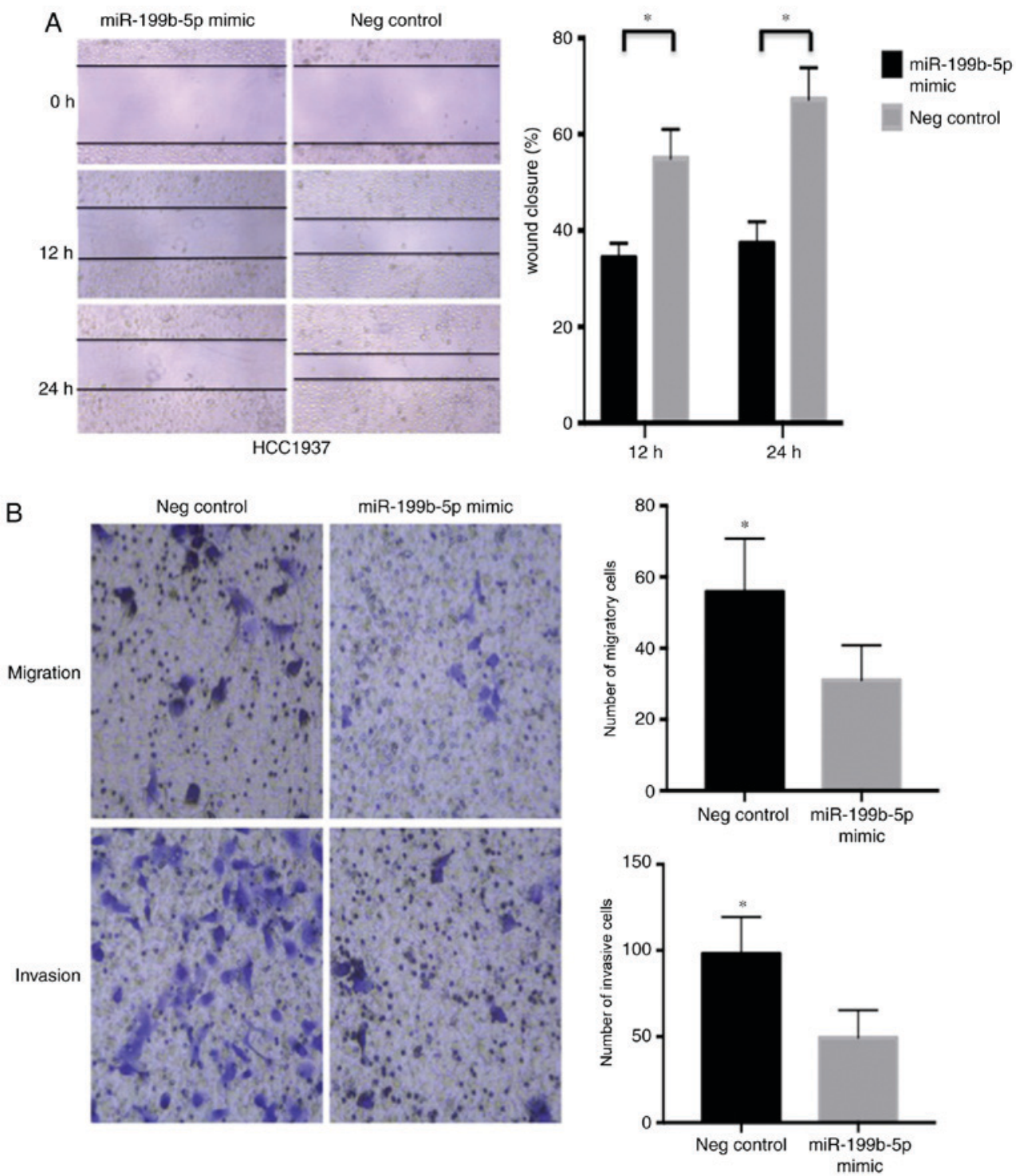

Figure 2. Upregulation of miR-199b-5p inhibits cell migration and invasion in HCC1937 cells. (A) Representative images of miR-199b-5p transfected HCC1937 cell monolayer wound healing following 12 and $24 \mathrm{~h}$ (magnification, x100). (B) Compared with the negative control group, the migration of HCC1937 was decreased following transfection with the miRNA-199b-5p mimic. miR-199b-5p-transfected HCC1937-cell invasion and migration were significantly inhibited compared with the negative control group. ${ }^{*} \mathrm{P}<0.05$ vs. negative control. miR, microRNA; neg, negative.
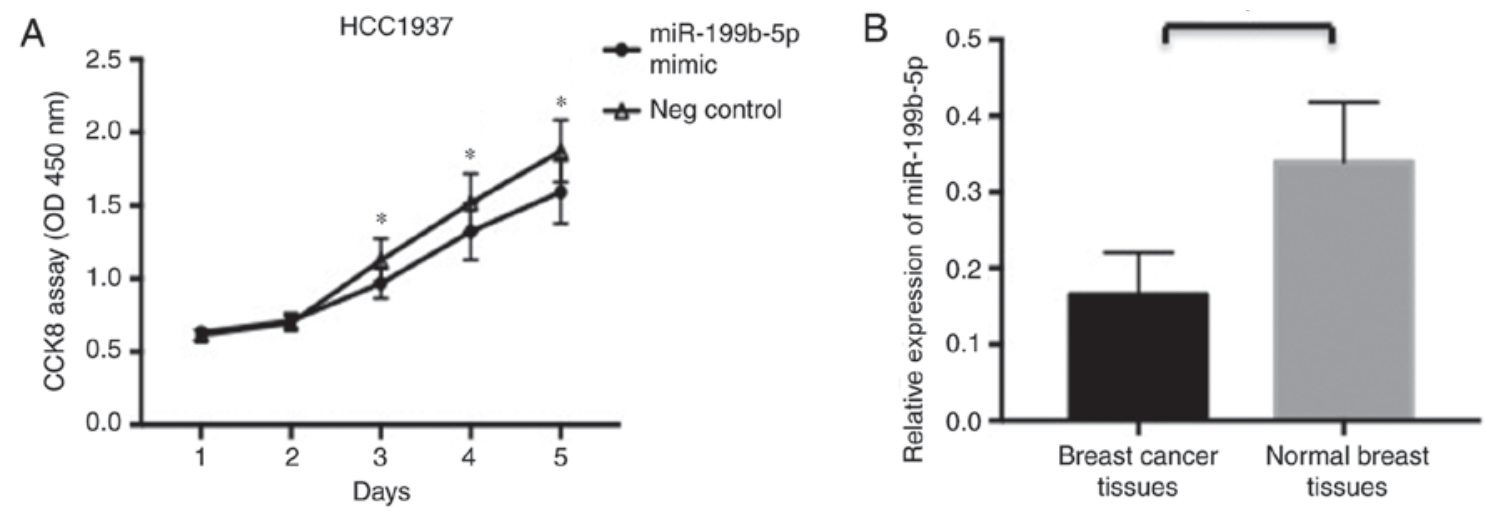

Figure 3. Upregulation of miR-199b-5p inhibits proliferation of HCC1937 cells and expression levels of miR-199b-5p in breast cancer tissues. (A) Compared with the negative control group, the proliferative ability of $\mathrm{HCC} 1937$ cells were significantly inhibited. (B) Compared with normal breast tissue, the expression of miR-199b-5p in cancer tissues was significantly reduced. " $\mathrm{P}<0.05$ vs. negative controls and normal cancer tissue. miR, microRNA; OD, optical density; neg, negative.

clinicopathological characteristics of miR-199b-5p in tissues were recorded. The results demonstrated that miR-199b-5p expression in breast cancer tissue was significantly reduced compared with normal breast tissue. This is congruent with 
A

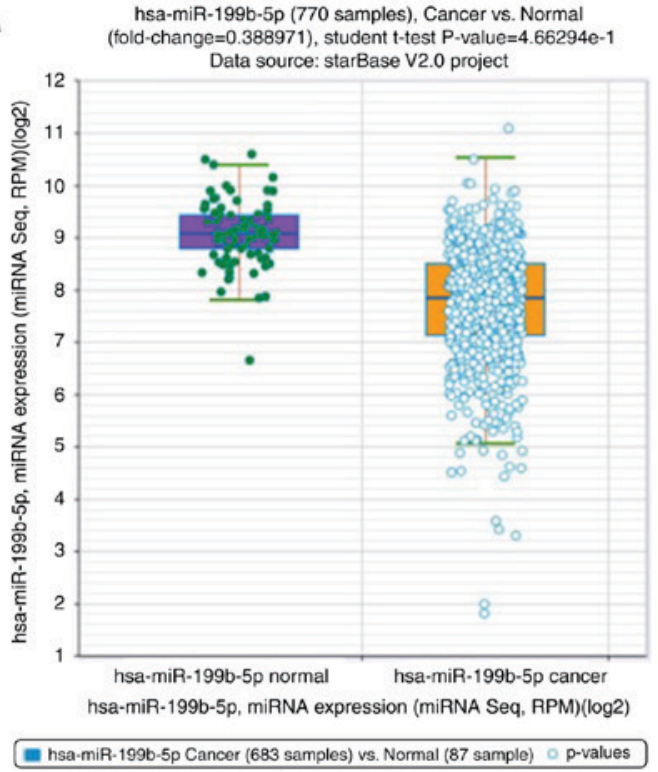

B DDR1 (1106 samples), Cancer vs. Normal (fold-change=1.858901),

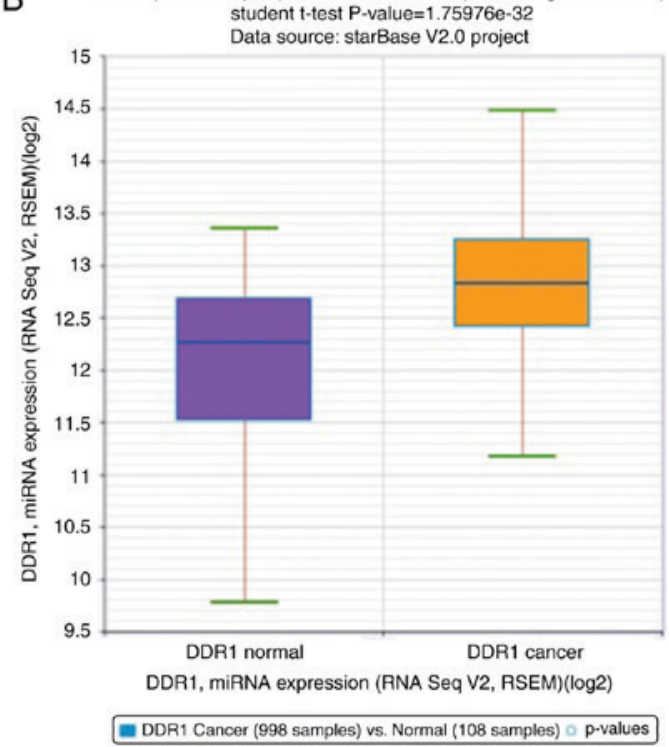

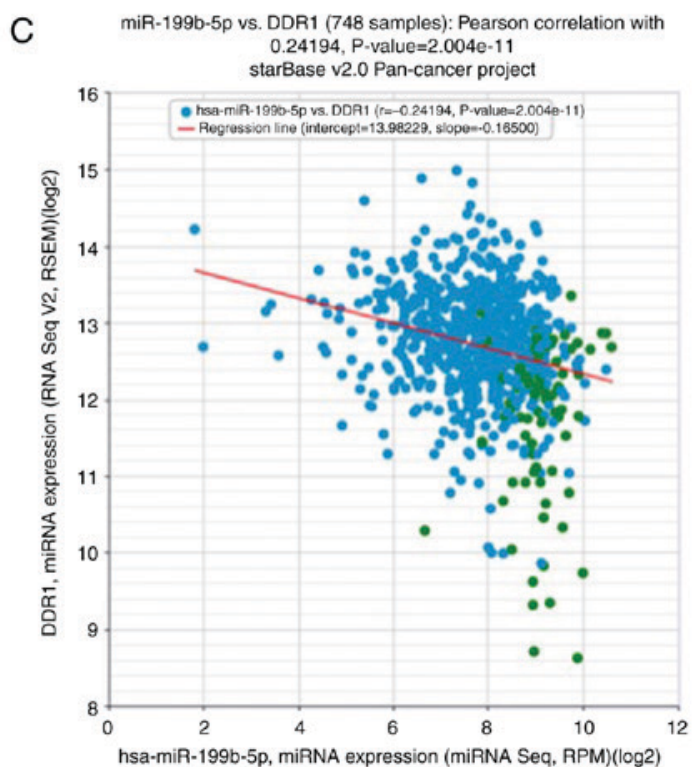

Figure 4. The expression of miR-199b-5p and DDR1, and the association between these genes in breast cancer tissue was anlaysed using public databases (http://starbase.sysu.edu.cn). (A) Compared with normal breast tissue, the expression of miR-199b-5p in cancer tissues was significantly reduced and (B) the expression of DDR1 was significantly upregulated. (C) The expression of miR-199b-5p was negatively correlated with DDR1. miR, microRNA; DDR1, discoidin domain receptor tyrosine kinase 1; seq, sequence; has, homo sapiens.

the result obtained from public databases. Furthermore, no statistically significant differences were according to age, tumor size or TNM stage. However, this may be a result of the relatively small sample size. Nevertheless, the loss of expression of miR-199b-5p in breast cancer may contribute to its carcinogenesis and progression.

DDR1 is a class of collagen receptor, expressed on various human leukocytes, including neutrophils, monocytes, lymphocytes and podocytes (9). DDR1 is also overexpressed in a variety of epithelial malignancies, including ovarian, breast, endometrial, esophageal and lung cancer (10-15). Of note, a previous study demonstrated that a DDR $1^{\text {Low }} / \mathrm{DDR} 2^{\text {High }}$ protein profile is associated with TNBC and may be used to identify invasive carcinomas with a poor prognosis (20). High DDR1 expression may also serve a role in breast cancer cell proliferation and/or survival $(21,22)$. A previous study has also indicated that the inhibition of DDR1 expression significantly enhances breast cancer cell chemosensitivity to genotoxic drugs (16). Furthermore, Valencia et al (15) demonstrated that the inhibition of DDR1 decreased cell survival, homing and colonization in lung cancer bone metastasis. Additionally, DDR1 overexpression promoted the migration and invasion of hepatocellular carcinoma and glioma cells $(17,18)$. Shen et al (19) also revealed that the decreased expression of miR-199a-5p in hepatocellular carcinoma contributed to increases in cell invasion via the functional deregulation of DDR1 activity. In the current study, the expression of DDR1 in patients with breast cancer were retrieved from a public database. Compared with normal breast tissue, the expression of DDR1 in breast cancer tissues was significantly upregulated. Thus, DDR1 may contribute to disease aggressiveness in a subset of invasive carcinomas. 
Table I. Comparative analysis of patient clinicopathological characteristics and miR-199b-5p in breast cancer tissue samples.

\begin{tabular}{|c|c|c|c|c|c|}
\hline \multirow[b]{2}{*}{ Characteristics } & \multirow[b]{2}{*}{ No. } & \multicolumn{2}{|c|}{ miR-199b-5p } & \multirow[b]{2}{*}{$\chi^{2}$} & \multirow[b]{2}{*}{ P-value } \\
\hline & & High & Low & & \\
\hline \multicolumn{6}{|l|}{ Age (years) } \\
\hline$<50$ & 10 & 2 & 8 & 0.014 & 0.906 \\
\hline$\geq 50$ & 9 & 2 & 7 & & \\
\hline \multicolumn{6}{|l|}{ Tumor size } \\
\hline$<3.0 \mathrm{~cm}$ & 10 & 3 & 7 & 1.017 & 0.313 \\
\hline$\geq 3.0 \mathrm{~cm}$ & 9 & 1 & 9 & & \\
\hline \multicolumn{6}{|l|}{ ER } \\
\hline$<10 \%$ & 7 & 1 & 6 & 0.305 & 0.518 \\
\hline$\geq 10 \%$ & 12 & 3 & 9 & & \\
\hline \multicolumn{6}{|l|}{ PR } \\
\hline$<10 \%$ & 8 & 2 & 6 & 0.130 & 0.719 \\
\hline$\geq 10 \%$ & 11 & 2 & 9 & & \\
\hline \multicolumn{6}{|l|}{ Ki67 } \\
\hline$<10 \%$ & 7 & 3 & 4 & 3.170 & 0.075 \\
\hline$\geq 10 \%$ & 12 & 1 & 11 & & \\
\hline \multicolumn{6}{|l|}{$\begin{array}{l}\text { Lymph node } \\
\text { metastasis }\end{array}$} \\
\hline Positive & 7 & 1 & 6 & 0.305 & 0.518 \\
\hline Negative & 12 & 3 & 9 & & \\
\hline \multicolumn{6}{|l|}{ TNM stage } \\
\hline I & 5 & 0 & 5 & 1.953 & 0.377 \\
\hline II & 8 & 2 & 6 & & \\
\hline III & 6 & 2 & 4 & & \\
\hline
\end{tabular}

TNM, Tumor-Node-Metastasis; ER, estrogen receptor; PR, progesterone receptor.

In conclusion, the present study demonstrated that miR-199b-5p directly modulates DDR1 expression by binding to the 3'UTR of DDR1. The overexpression of miR-199b-5p in HCC1937 cells also significantly reduced the expression of DDR1. Furthermore, the luciferase activity of DDR1 3'UTR-luc-WT was decreased upon transfection with miR-199b-5p mimics compared with control groups. However, no marked changes in luciferase activity were observed in the DDR1 3'UTR-luc-MUT group. The association of DDR1 and miR-199b-5p in breast cancer tissue was analyzed using public databases. The results demonstrated that the expression of miR-199b-5p is negatively associated with that of DDR1. The present study hypothesized that miR-199b-5p may inhibit the proliferation, migration and invasion of TNBC cells by inhibiting the expression of DDR1. Thus, DDR1 signaling may provide a novel target for the therapeutic intervention of TNBC in the future. However, few studies have assessed the regulation of miR-199b-5p, which should be further explored. Furthermore, the gene targeted by miR-199b-5p in TNBC, perhaps DDR2, requires determination in future studies. Due limitations of the present study, further analysis of the
A
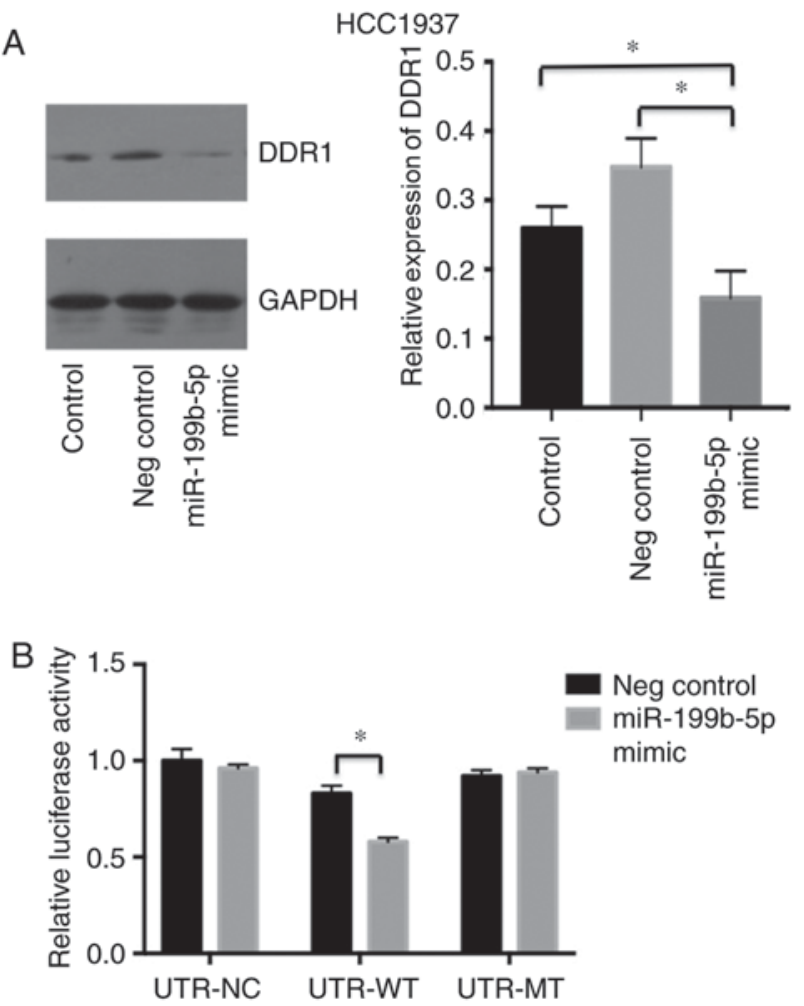

Figure 5. DDR1 is a novel direct target gene of miR-199b-5p. (A) The overexpression of miR-199b-5p in HCC1937 breast cancer cells significantly reduced the expression of DDR1. (B) The luciferase activity of DDR1 3'UTR-luc-WT was decreased following transfection with miR-199b-5p mimics when compared with the control groups. However, no marked changes in the luciferase activity of DDR1 3'UTR-luc-MUT were demonstrated. ${ }^{\text {* }} \mathrm{P}<0.05$. miR, microRNA; DDR1, discoidin domain receptor tyrosine kinase 1; UTR, untranslated region; WT, wild type; MUT, mutant; NC, negative control; Neg, negative.

association between miR-199b-5p expression, clinicopathological characteristics and the prognosis of patients with breast cancer is required, using a larger sample size. The results of the present study indicated that miR-199b-5p may serve a primary role in cancer suppression, potentially acting as a prognostic biomarker for breast cancer and potentially providing a novel approach for the management of TNBC.

\section{Acknowledgements}

The authors would like to thank Professor Ceshi Chen from the Kunming Institute of Zoology (Kunming, China) for the donation of Human breast cancer (HCC1937) and mammary gland (MCF-10A) cell lines.

\section{Funding}

This research was supported by the Initial Scientific Research Fund of Doctors from the Tumor Hospital of Yunnan Province (grant no. 201509) and Internal Research Projects of Yunnan Provincial Medicine and Health Units (grant no. 2016NS078).

\section{Availability of data and materials}

The datasets used and/or analyzed during the current study are available from the corresponding author on reasonable request. 


\section{Authors' contributions}

DL conceived and designed the present study; AW performed all in vitro experiments and the luciferase reporter assay, and drafted the manuscript; YC performed PCR and western blotting; YFL performed the statistical analysis; and YL critically revised the manuscript for important intellectual content and was involved in analysing and interpreting of data.

\section{Ethics approval and consent to participate}

The present study was approved by the Ethics Committee of Kunming Medical University (Kunming, China). Written informed consent was obtained from all patients.

\section{Patient consent for publication}

All patients provided consent for publication of this manuscript.

\section{Competing interests}

The authors declare that they have no competing interests.

\section{References}

1. Bartel DP: MicroRNAs: Genomics, biogenesis, mechanism, and function. Cell 116: 281-297, 2004.

2. Zeng H, Zhang Z, Dai X, Chen Y, Ye J and Jin Z: Increased expression of microRNA-199b-5p associates with poor prognosis through promoting cell proliferation, invasion and migration abilities of human osteosarcoma. Pathol Oncol Res 22: 253-260, 2016.

3. Fang C, Zhao Y and Guo B: MiR-199b-5p targets HER2 in breast cancer cells. J Cell Biochem 114: 1457-1463, 2013.

4. Fang C, Wang FB, Li Y and Zeng XT: Down-regulation of miR-199b-5p is correlated with poor prognosis for breast cancer patients. Biomed Pharmacother 84: 1189-1193, 2016.

5. Wu X, Weng L, Li X, Guo C, Pal SK, Jin JM, Li Y, Nelson RA, $\mathrm{Mu} \mathrm{B}$, Onami $\mathrm{SH}$, et al: Identification of a 4-microRNA signature for clear cell renal cell carcinoma metastasis and prognosis. PLoS One 7: e35661, 2012.

6. Favreau AJ, McGlauflin RE, Duarte CW and Sathyanarayana P: miR-199b, a novel tumor suppressor miRNA in acute myeloid leukemia with prognostic implications. Exp Hematol Oncol 5: 4, 2016

7. Wang C, Song B, Song W, Liu J, Sun A, Wu D, Yu H, Lian J, Chen L and Han J: Underexpressed microRNA-199b-5p targets hypoxia-inducible factor- $1 \alpha$ in hepatocellular carcinoma and predicts prognosis of hepatocellular carcinoma patients. J Gastroenterol Hepatol 26: 1630-1637, 2011.

8. Navrátil J, Fabian P, Palácová M, Petráková K, Vyzula R and Svoboda M: Triple negative breast cancer. Klin Onkol 28: 405-415, 2015 (In Czech).

9. Yoshimura T, Matsuyama W and Kamohara H: Discoidin domain receptor 1: A new class of receptor regulating leukocyte-collagen interaction. Immunol Res 31: 219-230, 2005.

10. Turashvili G, Bouchal J, Baumforth K, Wei W, Dziechciarkova M, Ehrmann J, Klein J, Fridman E, Skarda J, Srovnal J, et al: Novel markers for differentiation of lobular and ductal invasive breast carcinomas by laser microdissection and microarray analysis. BMC Cancer 7: 55, 2007.
11. Neuhaus B, Bühren S, Böck B, Alves F, Vogel WF and Kiefer F: Migration inhibition of mammary epithelial cells by Sky is blocked in the presence of DDR1 receptors. Cell Mol Life Sci 68: 3757-3770, 2011.

12. Quan J, Yahata T, Adachi S, Yoshihara K and Tanaka K: Identification of receptor tyrosine kinase, discoidin domain receptor 1 (DDRl), as a potential biomarker for serous ovarian cancer. Int J Mol Sci 12: 971-982, 2011.

13. Colas E, Perez C, Cabrera S, Pedrola N, Monge M, Castellvi J, Eyzaguirre F, Gregorio J, Ruiz A, Llaurado M, et al: Molecular markers of endometrial carcinoma detected in uterine aspirates. Int J Cancer 129: 2435-2444, 2011.

14. Nemoto T, Ohashi K, Akashi T, Johnson JD and Hirokawa K: Overexpression of protein tyrosine kinases in human esophageal cancer. Pathobiology 65: 195-203, 1997.

15. Valencia K, Ormazábal C, Zandueta C, Luis-Ravelo D, Antón I, Pajares MJ, Agorreta J, Montuenga LM, Martínez-Canarias S, Leitinger B and Lecanda F: Inhibition of collagen receptor discoidin domain receptor-1 (DDR1) reduces cell survival, homing, and colonization in lung cancer bone metastasis. Clin Cancer Res 18: 969-980, 2012.

16. Das S, Ongusaha PP, Yang YS, Park JM, Aaronson SA and Lee SW: Discoidin domain receptor 1 receptor tyrosine kinase induces cyclooxygenase-2 and promotes chemoresistance through nuclear factor-kappaB pathway activation. Cancer Res 66: 8123-8130, 2006.

17. Park HS, Kim KR, Lee HJ, Choi HN, Kim DK, Kim BT and Moon WS: Overexpression of discoidin domain receptor 1 increases the migration and invasion of hepatocellular carcinoma cells in association with matrix metalloproteinase. Oncol Rep 18: 1435-1441, 2007.

18. Ram R, Lorente G, Nikolich K, Urfer R, Foehr E and Nagavarapu U: Discoidin domain receptor-1a (DDR1a) promotes glioma cell invasion and adhesion in association with matrix metalloproteinase-2. J Neurooncol 76: 239-248, 2006.

19. Shen Q, Cicinnati VR, Zhang X, Iacob S, Weber F, Sotiropoulos GC, Radtke A, Lu M, Paul A, Gerken G and Beckebaum S: Role of microRNA-199a-5p and discoidin domain receptor 1 in human hepatocellular carcinoma invasion. Mol Cancer 9: 227, 2010

20. Toy KA, Valiathan RR, Núñez F, Kidwell KM, Gonzalez ME, Fridman R and Kleer CG: Tyrosine kinase discoidin domain receptors DDR1 and DDR2 are coordinately deregulated in triple-negative breast cancer. Breast Cancer Res Treat 150: 9-18, 2015.

21. Duncan JS, Whittle MC, Nakamura K, Abell AN, Midland AA, Zawistowski JS, Johnson NL, Granger DA, Jordan NV, Darr DB, et al: Dynamic reprogramming of the kinome in response to targeted MEK inhibition in triple-negative breast cancer. Cell 149: 307-321, 2012.

22. Marcotte R, Brown KR, Suarez F, Sayad A, Karamboulas K, Krzyzanowski PM, Sircoulomb F, Medrano M, Fedyshyn Y, Koh JLY, et al: Essential gene profiles in breast, pancreatic, and ovarian cancer cells. Cancer Discov 2: 172-189, 2012.

23. Livak KJ and Schmittgen TD: Analysis of relative gene expression data using real-time quantitative PCR and the 2(-Delta Delta C(T)) method. Methods 25: 402-408, 2001.

24. Chalmin F, Ladoire S, Mignot G, Vincent J, Bruchard M, Remy-Martin JP, Boireau W, Rouleau A, Simon B, Lanneau D, et al: Membrane-associated Hsp72 from tumor-derived exosomes mediates STAT3-dependent immunosuppressive function of mouse and human myeloid-derived suppressor cells. J Clin Invest 120: 457-471, 2010.

25. Ebert MS and Sharp PA: MicroRNA sponges: Progresss and possibilities. RNA 16: 2043-2050, 2010.

This work is licensed under a Creative Commons Attribution-NonCommercial-NoDerivatives 4.0 International (CC BY-NC-ND 4.0) License. 